\title{
Dantiscum septentrionis, occidentis et totius orbis hodie emporium frequentissimum. Ægidius Dickmans großes Panorama von Danzig von 1617
}

DOI: https://doi.org/10.26881/porta.2020.19.03

Vor etwas mehr als hundert Jahren wurde in der Königlichen Bibliothek in Stockholm ein bis dahin unbekanntes Panorama von Danzig gefunden. Es war mit anderen Städteansichten und Stadtplänen in einem Folianten vereint, der dem schwedischen Reichskanzler Magnus Gabriel de la Gardie (1622-1686) gehört hatte. Der Band enthielt mehrere sehr seltene Blätter und auch einige Unikate, wie das Bild von Danzig (Abb. 1). Die Danzigvedute ist 1617 datiert und mit dem vollen Namen des Künstlers, Aegidius Dickman, signiert $^{1}$. Sie ist von sieben Platten gedruckt worden und mit einer Länge von $258 \mathrm{~cm}$ außergewöhnlich groß. Eine in lateinischer und deutscher Sprache verfasste Beschreibung der Stadt ergänzt das Panorama. 1979 haben Eckhard Jäger und Ernst Bahr die Vedute zusammen mit den 14 Ansichten von Danzig, die Dickman ebenfalls 1617 herausgab, einem breiteren Lesekreis vorgestellt ${ }^{2}$. Bahr erläutert die topographischen Aspekte des Panoramas und der einzelnen Ansichten und stellt auch die wenigen bekannten Fakten zu Dickmans Biographie zusammen. Auf diese Publikation bauen die folgenden Bemerkungen auf.

Dickman bezeichnet sich in der Signatur als Danziger und gibt an, den Stich selbst zu verlegen: Egidius Dickman Gedanensis Fecit et Excudebat 1617. Vermutlich hat er das Panorama auch in Danzig herausgegeben³. Wir kennen aber weder seine Lebensdaten noch seinen beruflichen Werdegang. Gesichert ist

Isak Collijn, Magnus Gabriel de la Gardies samling af äldre stadsvyer och historiska planscher i Kungl. Biblioteket (Kungl. bibliotekets handlingar 35. Årsberättelse för år 1912), Stockholm 1915, S. 57f., Nr. 163, Tafel 12.

2 Eckhard Jäger (ed.), Egidius Dickmann und das Danziger Stadtbild um 1617 (mit Einführung und Kommentaren von Ernst Bahr), Lüneburg 1979, hier auch der vollständige Text der Beschreibung der Stadt.

3 Herkunft und Alter des Papiers sind nicht bekannt. Es wurde leider nicht untersucht, bevor die Blätter Anfang des vorigen Jahrhunderts auf einen Stoffträger aufgezogen wurden. Freundliche Mitteilung von Malin Joakimsson, Kungl. Biblioteket. 


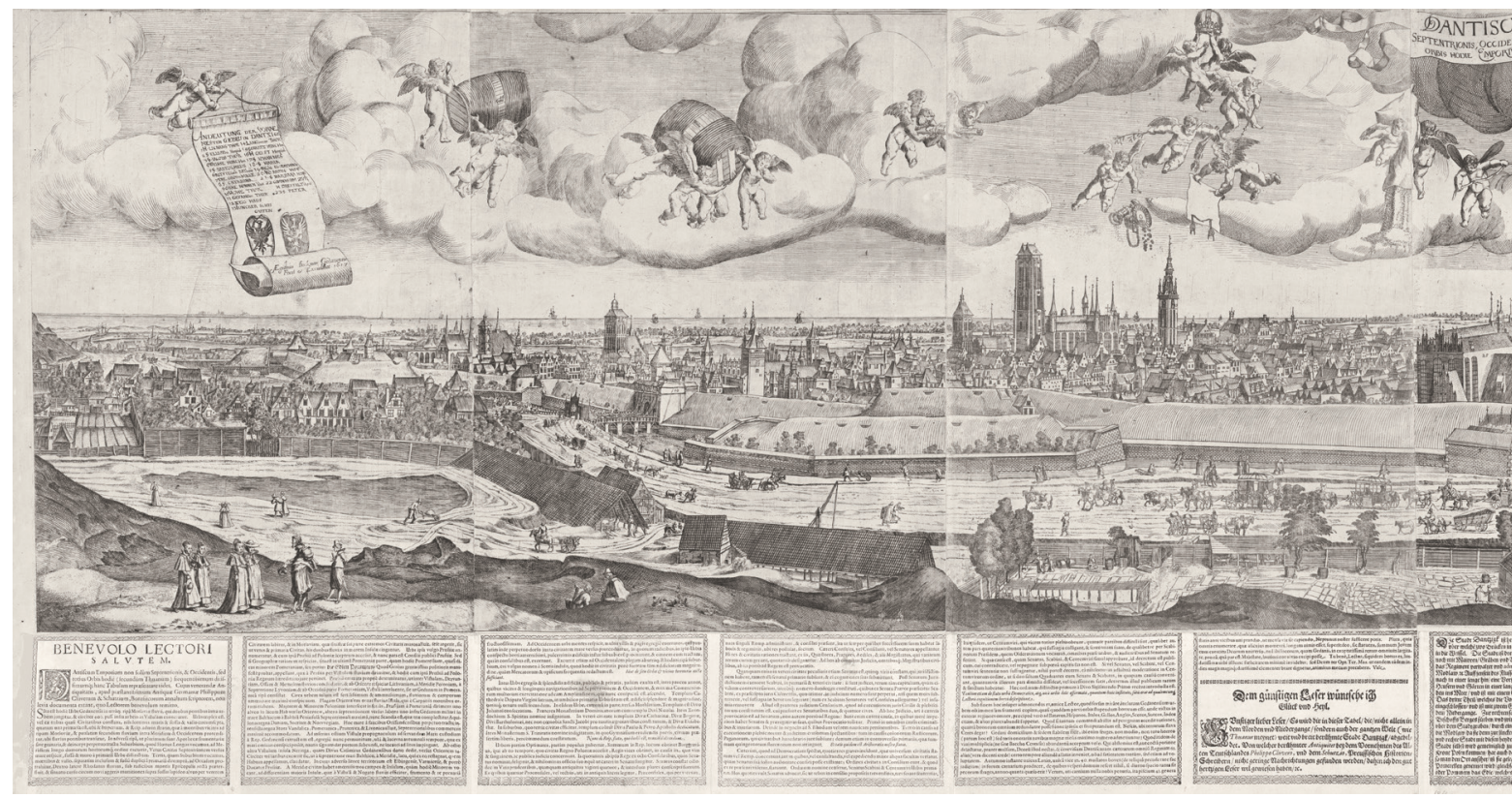

Abb. 1. Ægidius Dickman, Panorama von Danzig, 1617, Radierung (Stockholm, Kungl. Biblioteket)

nur, dass er sich 1624 in den Niederlanden aufhielt ${ }^{4}$. Die stilistische Nähe seiner Arbeiten zu den Radierungen holländischer Künstler wie Jan van de Velde und Claes Jansz. Visscher legt die Vermutung nahe, dass er dort auch seine künstlerische Ausbildung erhalten hat. Er kann sich aber natürlich auch an einem anderen Ort an Arbeiten von ihnen geschult haben. Außer der Vedute von 1617 und der genannten Folge von Danziger Ansichten ist heute nur noch eine 1618 datierte Radierung nachweisbar, „Besuch der Bauern bei dem Rechtsanwalt“, eine humoristische Genreszene in holländischem Stil 5 .

Die Idee, ein Panorama von Danzig in dieser monumentalen Größe herzustellen und es mit einem Titel zu versehen, der behauptet, die Stadt sei die meistbesuchte Handelsstadt der ganzen Welt, zeugt gleichermaßen vom Stolz des Künstlers auf seine Stadt wie von seinem persönlichen Ehrgeiz. Sie sagt aber auch etwas über das Selbstbewusstsein der Stadt selbst aus. Die prachtvollen

4 Ende März 1624 schreibt Dickman aus Haarlem an den Danziger Rat und bedankt sich für empfangene Unterstützung. Er verbindet den Dank mit der Versicherung seiner Bereitschaft, der Vaterstadt immer zu Diensten sein zu wollen, wenn ihm nur die nötigen Mittel dazu nicht fehlten. Schon im selben Jahr scheint sich ihm die Gelegenheit dazu geboten zu haben, als der Rat einen Vertrauensmann in den Niederlanden beauftragte, Dickman eine Anstellung als Ingenieur anzubieten. $\mathrm{Ob}$ er dieses Angebot angenommen hat, wissen wir nicht. Jäger, Egidius Dickmann, S. 12.

5 Braunschweig, Herzog Anton Ulrich-Museum (Inv. Nr. Aeg. Dickmann AB 3.15); Hollstein's German Etchings, Engravings and Woodcuts, 1400-1700, vol. VI, 1960, Nr. 16 führt das 1618 datierte, $Æ D$ schul. signierte und 26,3 ×30,6 cm große Blatt auf, ohne einen Standort anzugeben. 


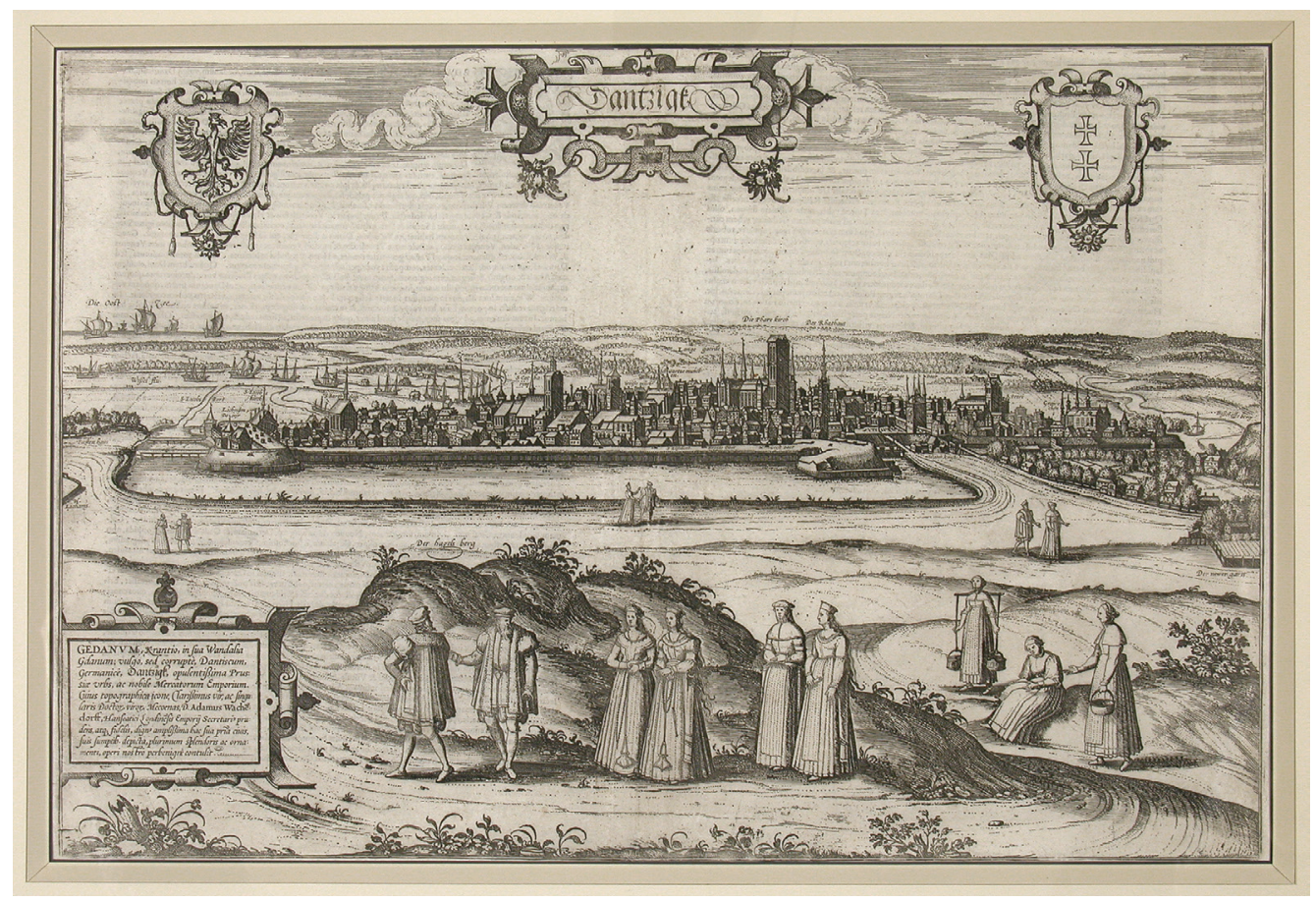

Abb. 2. Georg Braun, Frans Hogenberg, Danzig, 1575 (Polona - Polnische Digitale Bibliothek)

Vermutlich stellte Dickman seine Drucke in eigener Regie in Danzig her. Das große Panorama war jedoch nicht nur für den heimischen Markt bestimmt, es sollte Danzigs Ruhm in Europa verbreiten. Wie schwer es aber war, ohne die Unterstützung eines erfahrenen Verlegers auf dem internationalen Markt zu bestehen, musste er bald erfahren. Schon 1618 hat der in Amsterdam tätige Verleger Pieter van der Keere (1571-um 1646) die Vedute kopiert und veröffentlicht, ohne Dickmans Urheberschaft anzugeben (Abb. 4) ${ }^{7}$. Van der Keeres Kopie ist kleiner als Dickmans Original $(42,4 \times 212,5 \mathrm{~cm})$ und von vier Platten gedruckt. Damit passte Pieter van der Keere den Druck an die Konventionen des Graphikmarktes an. Die zahlreichen großformatigen Stadtansichten, die gerade in den ersten Jahrzehnten nach 1600 in Amsterdam produziert wurden, sind in der Regel von vier Platten gedruckt worden, was bei der üblichen Breite der Kupferplatten eine Länge von etwa $200-210 \mathrm{~cm}$ ergibt. Die Kopie gibt ihr Vorbild mit einigen Veränderungen wieder. Die

Hollstein's Dutch and Flemish Etchings, Engravings and Woodcuts, c. 1450-1700, vol. 9, 1953, S. 233 führt die Vedute nicht auf; Bert van't Hoff, Grote Stadspanorama's, gegraveerd in Amsterdam sedert 1609, In: „Jaarboek van het Genootschap Amstelodamus“ 47, 1955, S. 81-131; Meyer, Die Danziger Stadtvedute, S. 27f.; Von Danzig nach Riga. Ansichten, Stadtpläne und Landkarten von Ost- und Westpreußen, Danzig und dem Baltikum aus der graphischen Sammlung des Germanischen Nationalmuseums, Nürnberg o.J., Nr. 30. 


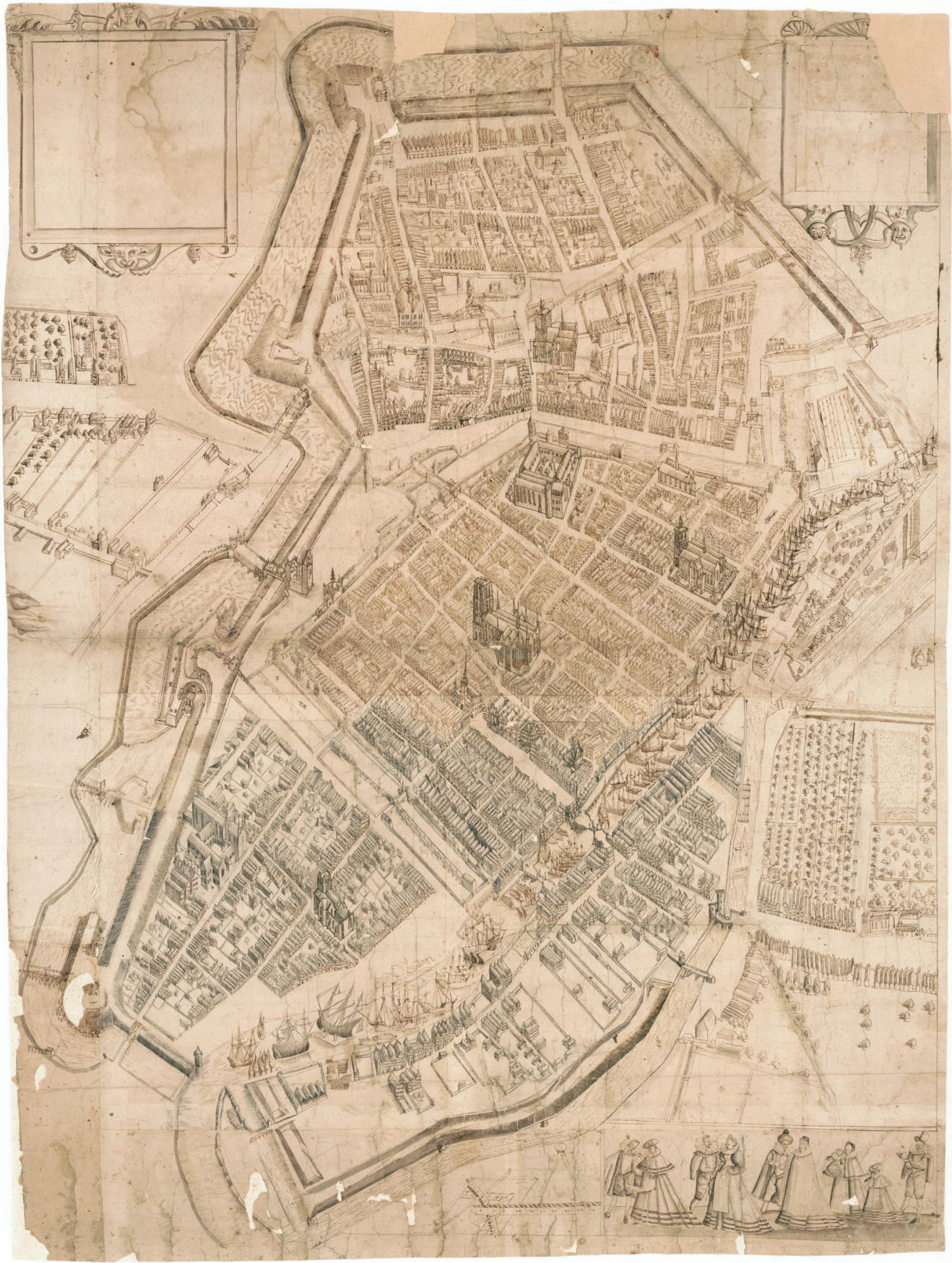

Abb. 3. Anton Möller (?), Danzig aus der Vogelperspektive, Zeichnung, um 1600 (Stockholm, Krigsarkivet: Utländska stads- och fästningsplaner, Tyskland, Danzig, nr 13) 


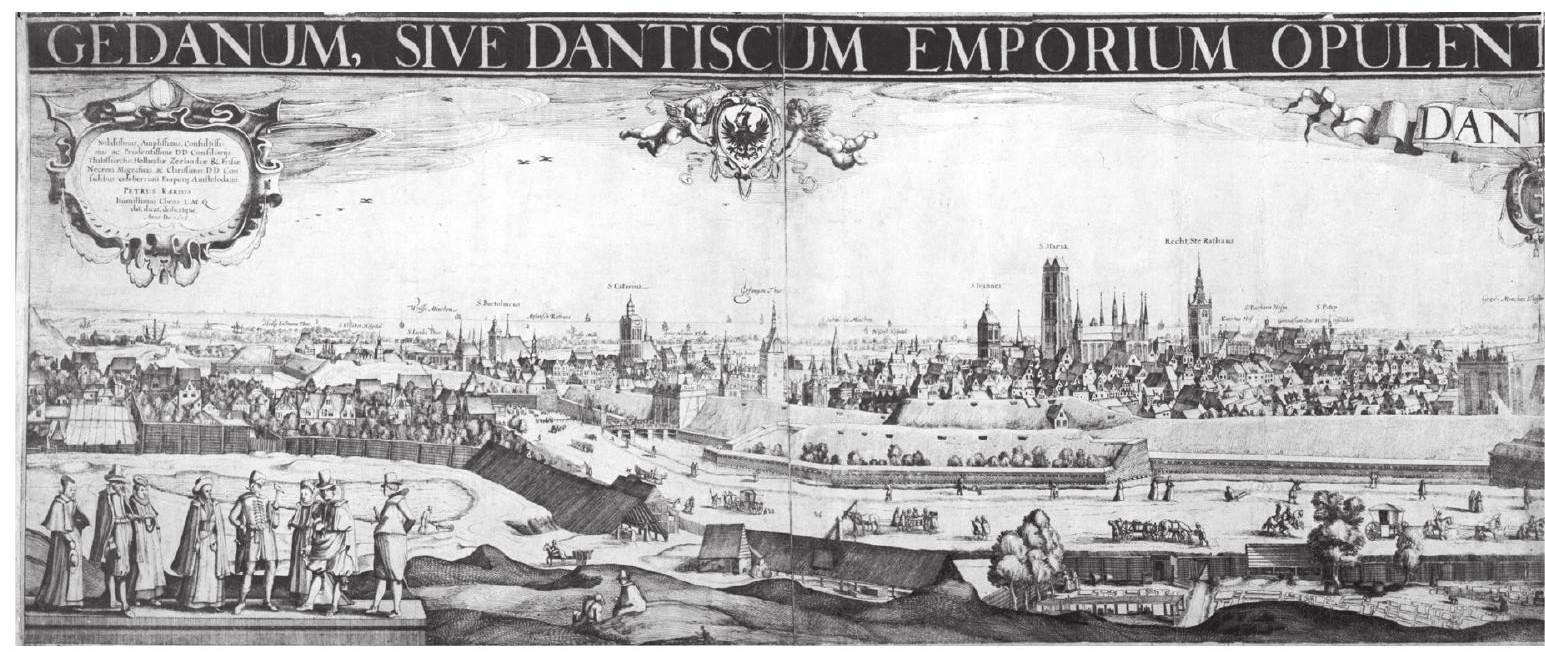

Abb. 4. Pieter van der Keere, Panorama von Danzig, 1618, Freie Kopie nach Dickman. Radierung (Nürnberg, Germanisches Nationalmuseum)

vielen Motive, die auf Dickmans Darstellung den Himmel beleben, wurden weggelassen und durch Wappen und Kartuschen mit Widmung und Signatur ersetzt. Van der Keere hat auch Dickmans großspurigen Titel abgeschwächt; er klingt aber auch hier immer noch recht stolz: GEDANUM, SIVE DANTISCUM EMPORIUM OPULENTISSIMUM, AMPLISSIMUM ET VENUSTISSIMUM (Die sehr reiche, große und schöne Handelsstadt Danzig). Bei den Figuren im Vordergrund hat der Kopist sich gegenüber der Vorlage recht frei verhalten. Die auffälligste Abweichung betrifft die Figuren links im Vordergrund. Van der Keere hat ihre Zahl von sechs auf acht erhöht und sie auf eine Art Postament gestellt. Damit sind sie, ähnlich wie die auf den Stadtansichten von Braun und Hogenberg üblichen Trachtenfiguren, aus dem Zusammenhang mit den übrigen Staffagefiguren gelöst. Für die Bemühung des Kopisten um Authentizität mag sprechen, dass er das modisch gekleidete junge Paar rechts aus dem Titelblatt von Dickmans Folge von 14 Ansichten übernommen hat.

Für Dickman dürfte Pieter van der Keeres Kopie eine empfindliche finanzielle Einbuße bedeutet haben ${ }^{8}$. So ärgerlich dies für ihn gewesen sein muss, so eindeutig zeigt die schnell auf den Markt gebrachte Kopie aber auch, wie begründet seine Hoffnung gewesen war, mit der Vedute gute Geschäfte machen zu können. Danzig war aus der Sicht Westeuropas wirtschaftlich sehr wichtig geworden, und entsprechend groß dürfte auch dort das Interesse an topographischen Darstellungen der Stadt gewesen sein. Die Bedeutung Danzigs als

8 Wie Bahr (in Jäger, Æidius Dickmann, S. 12) vermutet, bezieht sich darauf wohl die in seinem Dankesbrief erwähnte „Gelickes Widerwertigkeit“, die ihm widerfahren sei. 


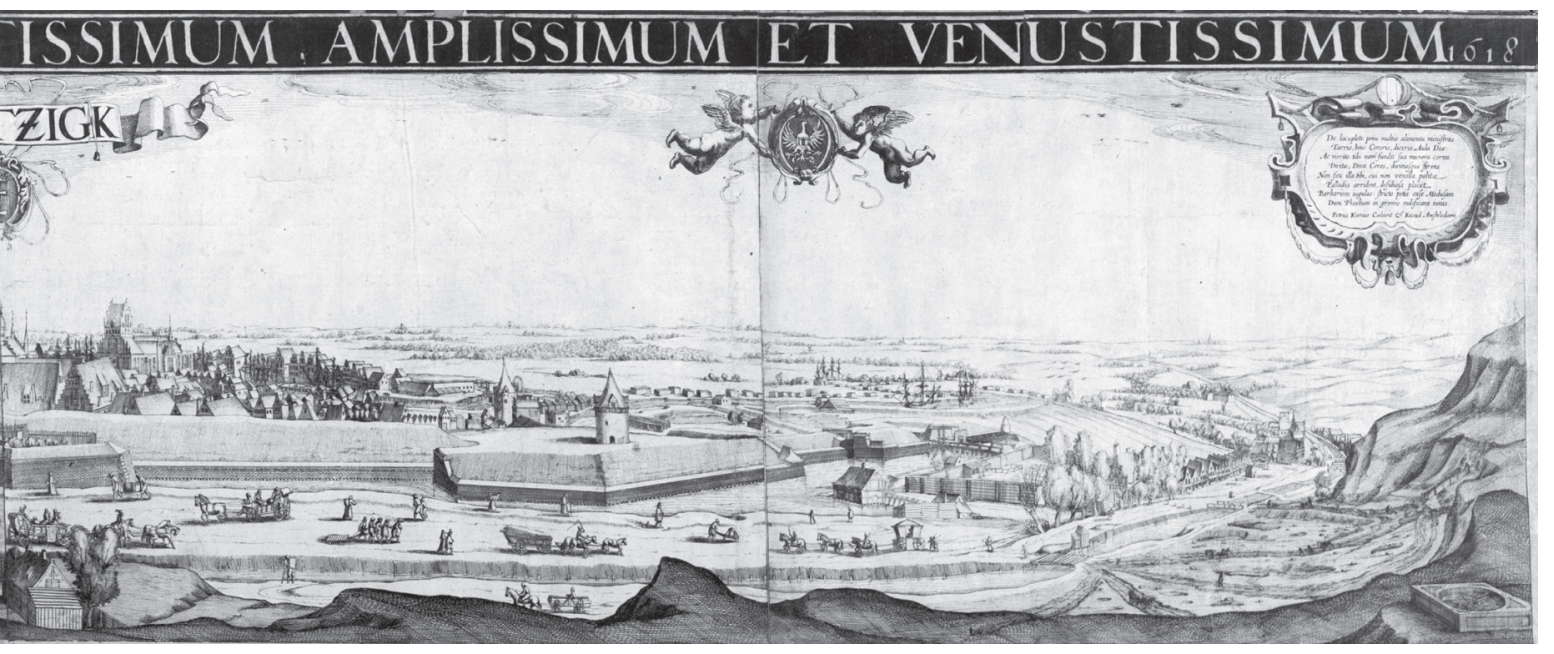

Export- und Importhafen ist, wie wir sehen werden, auf Dickmans Vedute sehr anschaulich dargestellt und wird in der Beschreibung mit selbstbewussten Worten gewürdigt.

Wegen der Konkurrenz aus dem Amsterdamer Verlagshaus sind vermutlich nur wenige Exemplare von Dickmans Vedute gedruckt worden. Ob das aber der einzige Grund dafür war, dass sie so selten geworden ist, sei dahingestellt. Es trifft nämlich auf alle bekannten großformatigen Stadtansichten zu, dass sie heute sehr rar sind. Ein Grund für den Schwund war vermutlich schon die Schwierigkeit, die großen Formate schonend aufzubewahren. Der Foliant in Stockholm stellt in dieser Hinsicht eine glückliche Ausnahme dar. Die großen Drucke scheinen oft als Wandschmuck gedient zu haben. Als solcher waren sie der Einwirkung von Licht und Schmutz meist schutzlos ausgesetzt und wurden früher oder später aussortiert, wenn sie nicht schlicht den wechselnden Moden zum Opfer fielen. Ein bis in unsere Zeit erhaltenes Beispiel für die Verwendung als Wandschmuck, sind die Stadtveduten, die seit dem 17. Jahrhundert als Supraporten über den Türen der nach ihnen benannten Zimmer auf Schloss Skokloster dienten ${ }^{9}$.

Betrachten wir nun Dickmans Vedute etwas genauer. Von einem fiktiven Aussichtspunkt auf dem Bischofsberg sehen wir die Stadt in ihrer vollen Länge von Nord bis Süd vor uns ausgebreitet. Über der Stadt, am Himmel,

9 Sie stammen aus dem Besitz des neben Magnus Gabriel de la Gardie größten schwedischen Sammlers des 17. Jahrhunderts, Carl Gustav Wrangel (1613-1676). Die Drucke, die sich inzwischen in einem schlechten Zustand befinden, wurden vor einigen Jahren abgenommen. Freundliche Mitteilung von Petri Tigercrona, Skokloster. 
Lars Olof der auf dem Blatt fast so viel Raum einnimmt wie die Darstellung der Stadt Larsson selbst, tummeln sich kleine Engel zwischen Titelbanderole, Wappen und anderen Hoheitszeichen. Sie tragen große Fässer und andere Gegenstände, die auf die Quellen des Reichtums der Stadt anspielen. Viel Raum nimmt auch der Vordergrund ein mit detailfreudigen Darstellungen der Vorstädte und des lebhaften Treibens auf der Hauptzufahrtstraße entlang des Raduniakanals. Unter dem Bild ist die Beschreibung der Stadt angebracht, in der ihre geographische Lage, ihre Gliederung in drei Städte, ihr „Regiment“, die wichtigsten Gebäude und die Quellen ihres Reichtums gerühmt werden. Solche ausführlichen, oft mehrsprachigen Beschreibungen fehlen unter den Stadtveduten selten, Dickmans Text zeigt, dass auch er auf eine internationale Verbreitung seines Druckes hoffte. Es mag ihm dabei auch daran gelegen haben, den Eindruck zu korrigieren, den der Text über Danzig bei Braun und Hogenberg vermittelte. Dort lesen wir eine skurrile Beschreibung der als Fischfang missverstandenen Bernsteingewinnung, in der die Danziger Bürger wie exotische Wilde auftreten ${ }^{10}$. Wie streng die Behörden darauf achteten, was publiziert wurde, zeigt sich darin, dass Dickman den Text vom Rat der Stadt vor dem Druck absegnen lie $\beta^{11}$. Aus seinem Schreiben lässt sich auch schließen, dass er die Vedute aus eigener Initiative gezeichnet hatte. Es handelte sich also nicht um einen städtischen Auftrag. Doch wird er nach vollendeter Arbeit eine Gratifikation erwartet haben. Das scheint damals der übliche Vorgang bei der Entstehung von Stadtveduten gewesen zu sein ${ }^{12}$. Die Initiative kam in der Regel von einem Künstler oder Verleger. Den Räten der betroffenen Städte waren solche Initiativen meist sehr willkommen, trugen die Veduten doch dazu bei, die Fama ihrer Stadt zu verbreiten ${ }^{13}$. Es gibt auch eine Reihe von Beispielen dafür, dass Veduten als diplomatische Geschenke genutzt wurden. So hat die Stadt Danzig, wie erwähnt, dem Gesandten von Venedig ein von Anton Möller gemaltes Panorama der Stadt überreicht, und

10 „Die Einwohner von Danzig laufen bei Tag und Nacht zum Meer und tragen Fischernetze bei sich, und sobald das Meer still wird, laufen sie nackt in das abebbende Meer und schöpfen mit ihren Fischernetzen vom Grund auf, was sie finden können [...] Und wenn sie das Netz eingeholt haben, laufen sie wieder ans Ufer, wo die Frauen warten, was sie Gutes mitbringen, und schütten die Netze aus, worauf die Frauen herausklauben, was gut ist“. Zitiert nach Georg Braun und Frans Hogenberg, Städte der Welt. Gesamtausgabe der kolorierten Tafeln 1572-1617, Hg. Stephan Füssel, Köln 2017, S. 182.

${ }_{11}$ Darum geht es in einem undatierten Schreiben an den Rat, das Bahr im Wortlaut wiedergibt (in Jäger, Ægidius Dickmann, S. 11).

12 Thomas Besing, Produktion und Publikum - Aspekte der Herstellung, Verbreitung und Rezeption frühneuzeitlicher Stadtdarstellungen, In: Wolfgang Behringer und Bernd Roeck, Das Bild der Stadt in der Neuzeit 1400-1800, München 1999, S. 94-100.

13 Ein bekanntes Beispiel dafür, wie sich ein Herrscher oder politisch Verantwortlicher bei einem Verleger dafür einsetzte, dass seine Stadt oder Region in einem topographischen Werk vorteilhaft berücksichtigt wurde, ist Heinrich Rantzau, auf dessen Betreiben die umfassende Präsentation von den Städten Holsteins in Braun und Hogenberg zurückzuführen ist. 
1612 schenkten die Generalstaaten dem türkischen Sultan Pieter van der Keeres Vedute von Amsterdam ${ }^{14}$.

Dass Dickman, wie seine Vorgänger, einen Aussichtspunkt auf den Höhen westlich der Stadt wählte, war angesichts der topographischen Situation fast selbstverständlich, obwohl von hier der Hafen nicht zu sehen war. Anders als Braun und Hogenberg stellte er die Stadt aber nicht vom Hagelsberg, also vom Nordwesten her dar, sondern von dem weiter südlich gelegenen Bischofsberg. Von hier aus war die Stadt gut zu überblicken, die Kirchen und die beiden Rathäuser waren deutlicher voneinander getrennt zu sehen, und im Osten, hinter der Stadt, konnte man den flachen Werder erblicken; inwiefern man auch die Ostsee und die Halbinsel Hela sehen konnte, wie uns Dickman glauben lassen will, sei dahingestellt.

Die Suggestion, dass die Stadt von einem bestimmten Standpunkt aus abgebildet worden sei, wird im Bild selbst thematisiert. Wir sehen links im Vordergrund einen Künstler sitzen, mit dem Zeichenblock auf dem Schoß. Neben ihm hockt ein einfach gekleideter Mann, der gerade vorbeigekommen zu sein scheint. Er schaut neugierig dem Zeichner zu und weist auf die Stadt (Abb. 1). Mit seiner Geste zeigt er, was der Künstler zeichnet; gleichzeitig fordert er uns auf, die vor unseren Augen ausgebreitete Stadt selbst zu bewundern und vielleicht auch, uns in der Phantasie in sie hineinzubegeben. Dass der Künstler von diesem Punkt aus die Stadt nicht so sehen konnte, wie sie auf dem Bild erscheint, unterstreicht nur, dass das Motiv ein Topos ist. In der Realität konnte die Stadt ohnehin nicht von nur einem Standpunkt aus erfasst werden, wollte man, wie Dickman, alle wichtigen Gebäude zu ihrem Recht kommen lassen.

Dass die Wallanlagen einen so beherrschenden Raum auf der Vedute einnehmen, war schon in den realen Gegebenheiten begründet. Danzig war bereits seit Langem eine stark befestigte Stadt, und gerade in den Jahrzehnten vor der Entstehung von Dickmans Vedute, waren neue Befestigungsanlagen nach modernsten Prinzipien gebaut worden. Das Bild der Stadt hinter den Wällen und Bastionen wird in Dickmans Zeichnung von den Türmen der Kirchen und den beiden Rathäusern geprägt. Dass die wichtigsten Gebäude und vor allem ihre Türme höher und monumentaler erscheinen als in der Realität, entspricht der üblichen Verfahrensweise der Zeichner solcher Ansichten.

Bemerkenswert differenziert ist die Darstellung der Umgebung der Stadt. Wir sehen nicht nur die Vorstädte Neugarten - unmittelbar vor dem Hohen

14 Van't Hoff, Grote Stadspanorama's, S. 94.; Meyer, Die Danziger Stadtvedute, S. 21, Georg Cuny, Danziger Kunst und Kultur im 16. und 17. Jahrhundert, Bd. 1, Frankfurt am Main 1910, S. 3. Die Müller zugeschreibene Zeichnung (Abb. 3. schwarze Tinte und Sepia) befindet sich in Krigsarkivet in Stockholm (Utländska stads- och fästningsplaner, Tyskland, Danzig, nr. 13). Sie misst $100 \times 73 \mathrm{~cm}$. Heinz Lingenberg schreibt sie Anton Möller zu und datiert sie überzeugend um 1600: Heinz Lingenberg, „Der älteste Grundrissplan Danzigs - ein Werk Anton Möllers?“, in: Unser Danzig 1-2, 1984; Ulla Ehrensvärd, Pellervo Kokkonen und Juha Nurminen, Mare Balticum. 2000 Jahre Geschichte der Ostsee, Helsinki 1996, S. $100 f$. 
Lars Olof Tor - und Schottland im Süden, sondern auch den flachen Werder im Osten, Larsson einen Teil des Weichsellaufs, den Waldstreifen an der Ostseeküste und am Horizont sogar die Halbinsel Hela. Dagegen hat Dickman im Bild keinen angemessenen Platz für die Speicherinsel gefunden. Sie wird zum größten Teil von den hohen Gebäuden zwischen der Marienkirche und dem Langen Markt verdeckt. Nur im Südosten, hinter der Peter und Paulskirche, sind Speichergiebel und Masten zu sehen, die auf diesen wirtschaftlich wichtigen Stadtteil aufmerksam machen. An die Bedeutung Danzigs als Hafen und Handelsstadt erinnern natürlich auch die vielen Schiffe auf der Ostsee und dem Fluss, die dem Hafen zustreben. Die Bedeutung der Stadt als Handelsplatz zu zeigen, ist aber vor allem der Zweck der Szenen am Himmel. Schon die Banderole mit dem Namen der Stadt schlägt das Thema an: DANTISCUM SEPTENTRIONIS, OCCIDENTIS ET TOTIUS ORBIS HODIE EMPORIUM FREQUENTISSIMUM (Danzig, heute der am meisten besuchte Handelsplatz im Norden, im Westen und in der ganzen Welt). Direkt unter diesem stolzen Spruch schütten zwei Engel einen Sack voller Korn über die Stadt aus (Abb. 5). Das ist ein prägnantes Bild für die wichtigste Quelle des Danziger Reichtums, den Getreidehandel. Direkt neben dieser Szene tragen zwei Engel ein Bündel von Büchern, Papierbögen, Mess- und Zeicheninstrumenten sowie einen Merkurstab, was daran erinnert, dass Danzig den Anspruch erhob, auch eine Heimstätte von Wissenschaft und Gelehrsamkeit zu sein. Wie an den großen Fässern zu sehen, die andere Engel heranschleppen, war Getreide nicht das einzige Handelsgut: „Oehl“, Wein, Salpeter (Salz), „Weudeasche“ (Pottasche) und Teer gehörten auch dazu, sowie Gewürze („Canel“ - riesige Zimtstangen), Ochsenhäute und Holz (eher klein geratene Bretter). Das Fass mit der Aufschrift „Oehl“ gibt in diesem Zusammenhang ein Rätsel auf (Abb. 6). Welches Öl wurde damals in solchen Mengen in den Ostseehäfen gehandelt? Der Umstand, dass das Fass direkt neben dem Weinfass dargestellt und von gleicher Größe und Machart wie dieses ist, erlaubt vielleicht die Vermutung, dass es sich gar nicht um Öl, sondern um Bier handelt. Bier war, wie aus der Beschreibung unter der Vedute hervorgeht, ein großer Handelsartikel in Danzig, und die Bezeichnung „Oehl“ für Bier ist im deutschsprachigen Ostseeraum nachweisbar, wenn auch nicht sehr geläufig. Vielleicht ist hier sogar ein besonderes, gutes, gehaltvolles Bier gemeint? In einer Quelle von 1590 heißt es immerhin „....ein gutes, rothes Bier, das rostocker Oehl genanndt“ (vgl. Anm. 15). Die Beschreibung unter der Vedute verstärkt noch den Eindruck des Überflusses, den uns die allegorischen Motive am Himmel vermitteln, und ist es wert, im Wortlaut zitiert zu werden:

„Zu letzt, lieber Leser, kan ich dich zu berichten keinen umbgang haben, dass die Stadt Dantzig, von wegen der grossen Menge des Korns, gleichsam eine kleine Weltscheure sey, aus welcher Korn in alle andere Länder, fürnemlich aber in Niederland, Spanien, Welschland, Franckreich, Engelland, Schottland, Schweden und Indien, auch in viel mehr andere Länder geführet wird, dass mann 


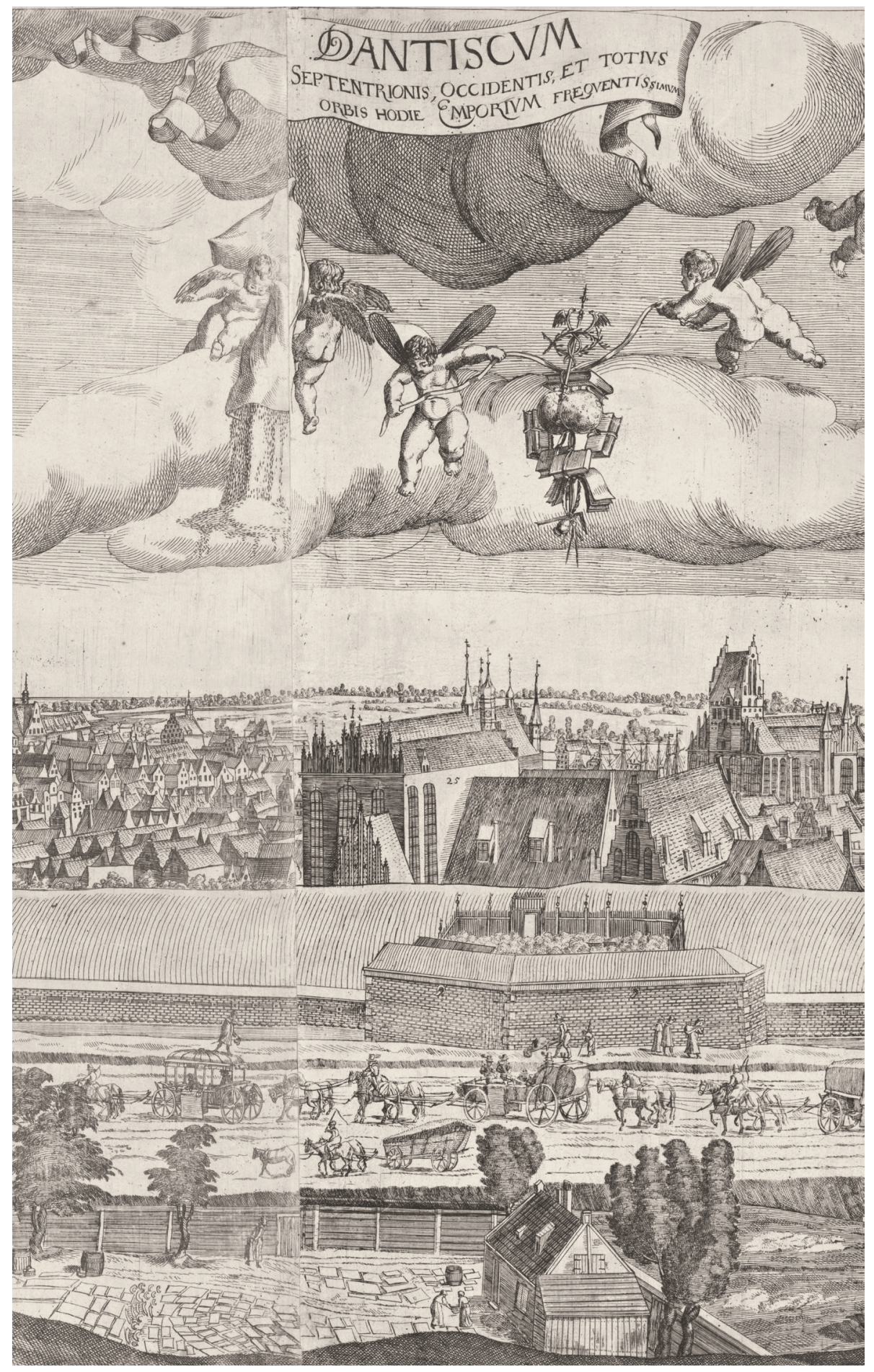

Abb. 5. Ægidius Dickman, Panorama von Danzig, Ausschnitt von Abb. 1 


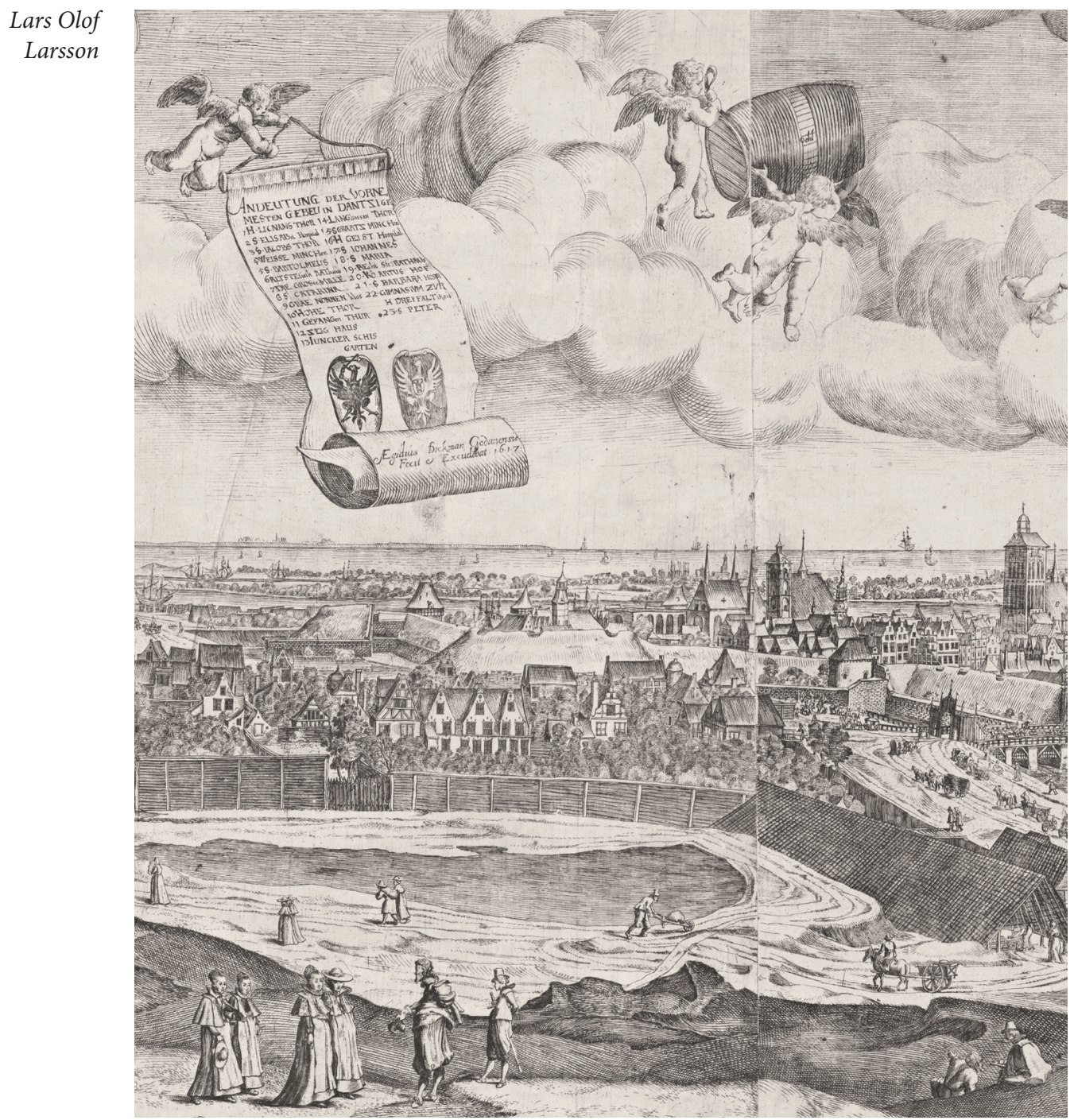

Abb. 6. Ægidius Dickman, Panorama von Danzig, Ausschnitt von Abb. 1

wohl sagen möchte, hie hette Ceres ihr einen bequemen Sitz bereitet, denn hie wird des lieben Getreides nicht ein Scheffel, nicht eine ganze Scheure voll [...] sondern gantze grosse Lastschiffe voll gemessen: welches ich auch von dem mannigfaltigen Weine (denn wo Ceres ist, wil Bacchus auch seyn) wil verstanden haben, dessen hie mehr als zwantzigerley verhanden. Sonsten soll man offt allhie sechzigerley Bier zu wege bringen können. Im Herbst bringet ein Fleischhauer des Morgens dreyssig oder wol viertzig geschlachtete Ochsen 
auffn Fleischmarckt, (wie viel des anderen Viehes sey, kannstu leicht bey dir selbst abmessen) davon ihrer keiner nichts auffn Abend wieder mit sich nach Hause bringet...".15

Wenden wir uns jetzt wieder dem Bild selbst zu. Unklar ist, ob der Becher, aus dem anscheinend Wein fließt, die Schüssel, aus der Konfekt herunterfällt, und die lange Kette auf das blühende Gewerbe der Danziger Gold-und Silberschmiede anspielen, oder ob sie, was wohl wahrscheinlicher ist, wie der Privilegienbrief und der Ölzweig als Teil der Wohltaten verstanden werden sollen, die der durch Krone und Zepter symbolisierte polnische König der Stadt gewährte. Von links oben lässt ein Engel eine im Winde wehende Schriftrolle herunter, auf der eine Liste der auf der Vedute gezeigten wichtigsten Gebäude, die Adler Preußens und Polens und die Signatur des Künstlers zu sehen sind. Die Verteilung der Handelsgüter am Himmel scheint auf die Topographie der Stadt abgestimmt zu sein. Pottasche und Teer befinden sich über dem Aschhof und dem Teerhof am Südende der Speicherinsel und die Bretter über dem Dielenplan, während die edleren Waren sich über dem Mottlauhafen vor dem Grünen Tor befinden.

Diese Motive waren Pieter van den Keere vermutlich nicht niveauvoll genug, vielleicht wirkten sie, wie die Überschrift auch, in Amsterdam einfach zu anmaßend. Wie dem auch sei, van der Keere hat sie jedenfalls durch symmetrisch angeordnete Kartuschen mit einer Widmung und den Wappen Danzigs, Preußens und Polens ersetzt.

Die Art, wie Dickman den Reichtum und die Bedeutung seiner Stadt herausstreicht, mag auf uns tatsächlich naiv wirken, gerade diese Motive tragen aber wesentlich zur Originalität der Darstellung bei und geben ihr eine Sonderstellung unter den Stadtveduten der Epoche. Die realistisch direkte, „volkstümliche“ Symbolsprache war der holländischen Kunst der Zeit übrigens ebenfalls nicht fremd. Sie stellt vielmehr, gerade in der ersten Hälfte des 17. Jahrhunderts, eines ihrer besonderen Merkmale dar. Nur herrschten auf dem international ausgerichteten Graphikmarkt striktere Konventionen; am Himmel über der Darstellung der Städte waren nur symmetrisch angeordnete Wappen und Kartuschen mit Schrift und keine zusätzlichen, frei erfundenen Motive, wie bei Dickman, üblich.

Dickmans Darstellung der einzelnen Gebäude, wie auch - und vor allem - die Wiedergabe von Beiwerk, etwa Brücken und Zäune, zeichnet sich durch Akribie

15 Jäger, Ægidius Dickmann, S. 41. Dass in der Beschreibung der Ausdruck „Bier“ ebenfalls verwendet wird, spricht nicht gegen die Vermutung, dass Dickman mit der Bezeichnung „Oehl“ Bier gemeint haben kann. Der Text wurde, wie berichtet, dem Rat zur Kontrolle vorgelegt und von diesem sicher auch sprachlich bearbeitet und entspricht nicht unbedingt Dickmans Ausdrucksweise. Als Beleg für den Gebrauch des Wortes „Oehl“ für Bier sei hier nur auf den Eintrag im Mecklenburgischen Wörterbuch (Bd. 5, Sp. 172) hingewiesen. Dort ein Beleg von 1590. Ich danke Professor Michael Elmentaler, Kiel, für diesen und andere Hinweise. 
Lars Olof und fachmännisches Verständnis aus. Besonders deutlich ist das an den aus relativ Larsson kleiner Entfernung gesehenen Gebäuden und Zäunen des Neuen Gartens zu beobachten. Dass der Neue Garten eine vornehme Vorstadt mit prächtigen Gebäuden war, ist nicht zu übersehen. Die Motive im Vordergrund geben einen Eindruck vom regen Verkehr und dem Gewerbefleiß vor den Wällen der Stadt. Großen Raum nimmt eine Anlage in der Mitte des Streifens ein. Sie stellt vermutlich eine Färberei dar und weist, wie unabsichtlich, auf die Sauberkeit des Wassers hin ${ }^{16}$. Große Laken sind an Leinen zum Trocknen aufgehängt und auf dem Boden liegen Tücher zum Bleichen ausgebreitet. Ein Tor zu einer Brücke über den Kanal steht offen und auf der Straße davor wird ein Wagen voller Säcke entladen. Die unten offenen Scheunen näher am Hohen Tor dürften Ziegelscheunen sein und daneben, direkt am Kanal, sehen wir die Dächer der Lohmühle und des Wasserwerks, von dem aus die Stadtbrunnen versorgt wurden. Ganz rechts im Bilde wird Sand abgebaut. Dieser wird vom steilen Hang auf einer Rutsche nach unten befördert, wo Männer mit Handkarren und Pferdewagen ihn aufladen und wegfahren. Auf der Straße verkehren in beide Richtungen Fußgänger, Fuhrwerke und elegante Equipagen. Genau in der Mitte des Bildes sehen wir eine vornehme Reisegesellschaft, die mit großem Gefolge dem Hohen Tor zusteuert. Links im Vordergrund schließlich werden zwei schön gekleidete Frauen von Kavalieren begrüßt; den Frauen folgen mit gebührendem Abstand zwei Mägde. Diese Figuren erinnern an die Trachtenfiguren, die Braun und Hogenberg ihren Stadtansichten mitzugeben pflegten, sind aber zu klein und zu wenig detailliert dargestellt, um Auskunft über die Danziger Kleidermode geben zu wollen. Etwas fremd wirkt in diesem Zusammenhang die mühlsteinartige Windrose rechts im Vordergrund, die dort wie zufällig abgelegt scheint.

Gleichzeitig mit der großen Vedute gab Dickman die bereits erwähnte Sammlung von Stadtansichten heraus. Auch wenn er die beiden Werke selbst nicht als zusammengehörend betrachtet haben sollte, liegt es nahe, sie so zu sehen. In den Stadtansichten konnte er wichtige Motive, die auf dem großen Prospekt keinen Platz fanden, detailliert darstellen. Dazu zählen die Festung Weichselmünde, der Mottlauhafen mit dem imposanten Grünen Tor, die Speicherinsel und bedeutende Plätze wie der Lange Markt. Bemerkenswert ist auch der Stadtplan, der die Folge abschließt.

In einer Neuauflage der Folge von 1625 sind die Blätter nummeriert. Betrachtet man sie in der vorgegebenen Reihenfolge, wird ein Besuch der Stadt suggeriert. Wir segeln an der Festung Weichselmünde vorbei und legen im Hafen an der Grünen Brücke an. Durch das Grüne Tor betreten wir den Langen Markt und durchqueren, an Artushof und Rechtstädtischem Rathaus vorbei, die Stadt

16 Im Gegensatz zur Mottlau führte die auf den Höhen bei Kartuzy entspringende Radunia sauberes und für die Wasserversorgung der Stadt unentbehrliches frisches Wasser. Curicke beschreibt fast poetisch das Flüsschen: „Es ist die Rodaun zwar ein kleiner, aber edler und sehr nützlicher Fluß, und ein sonderlich Kleinodt der Stadt Dantzig,... “. Reinhold Curicke, Der Stadt Danzig historische Beschreibung (1687), Hg. Siegfried Rosenberg, Hamburg 1979, S. 34. 
bis zum Stockturm und Hohen Tor. Inwiefern eine solche Lesart beabsichtigt war, ist allerdings fraglich. Die Reihenfolge der übrigen Blätter lässt jedenfalls keine solche Systematik erkennen. Vergleichen wir Dickmans Ansichten z. B. mit den Abbildungen in Reinhold Curickes Beschreibung der Stadt, fällt auf, dass Dickman nie einzelne Gebäude in den Mittelpunkt stellt. Auch Repräsentationsbauten wie die Rathäuser, der Artushof, das Grüne Tor oder das Zeughaus werden als Teile von ganzen Milieus gezeigt. Ebenso bemerkenswert ist, dass die Kirchen, die auf der großen Vedute die Silhouette der Stadt beherrschen, hier ganz fehlen. Als einziges einzelnes, freistehendes Gebäude steht nur die große Mühle im Zentrum einer Ansicht. Dazu passt, dass eine Mehrzahl der Ansichten dem Hafen und der Speicherinsel als dem wirtschaftlichen Herzstück der Stadt sowie dem für Handel und Gewerbe wichtigen Dominikplatz und dem Holzmarkt gewidmet sind. Dickman ging es vor allem darum, das Leben und Treiben im Hafen und in der Stadt - und nicht so sehr die einzelnen Gebäude - zu zeigen. Das macht sich auch darin bemerkbar, dass die Gebäude mit ihren überlängten Proportionen und variierenden Umrissen dem Gesamtduktus der Zeichnung untergeordnet werden. Vergleichbare Darstellungen von Plätzen und Gebäuden gab es damals vor allem von Rom und anderen italienischen Städten, aber auch von der Handelsmetropole Amsterdam. Das unmittelbare Vorbild für Dickman dürften Claes Jansz. Visschers 1611 datierte Ansichten von Amsterdam gewesen $\operatorname{sein}^{17}$.

Die Neuauflage von 1625 wurde von Visscher in Amsterdam herausgegeben. Die Blätter sind nummeriert und mit dem Monogramm Dickmans signiert: ED fe. Anno 1625. Es scheint also zu einer persönlichen Verbindung zwischen Dickman und Visscher gekommen zu sein. In diesem Zusammenhang erinnern wir uns an Dickmans Aufenthalt in Holland 1624. Er wird Kontakte mit den Amsterdamer Graphikverlegern geknüpft und dabei eingesehen haben, dass die Zusammenarbeit mit einem etablierten Verlag viel erfolgversprechender war als eine selbständige Unternehmung. Es ist nun auch interessant zu sehen, dass Visscher ebenfalls das große Panorama neu herausgegeben hat (Abb. 7) ${ }^{18}$. Die beiden einzigen heute bekannten Exemplare dieses Druckes enthalten aber, anders als die Blätter der 14 Ansichten, weder einen Hinweis auf Dickmans Urheberschaft noch eine Datierung. Die Frage ist also, ob Dickman an dieser Neuauflage überhaupt beteiligt war. Das ist aber wohl anzunehmen, so wie man vermuten kann, dass Visscher das Danzig-Panorama etwa gleichzeitig mit den 14 Ansichten herausgegeben hat. Die heute bekannten Drucke sind allerdings mit der Verlegersignatur Claes Jansz. Visscher junior versehen. Das deutet darauf, dass sie erst aus der Zeit nach etwa 1650 stammen, als Claes

17 Hollstein's Dutch and Flemish Etchings, Engravings and Woodcuts, c. 1450-1700, vol. 38, 1991, Nr. 144-147; Maria Simon, Claes Jansz. Visscher (unpublizierte Diss. Freiburg i. Br. 1958), Nr. $147-50$.

18 Hollstein's Dutch and Flemish Etchings, vol. 38, Nr. 132. Simon, Claes Jansz. Visscher, S. 217-219. 


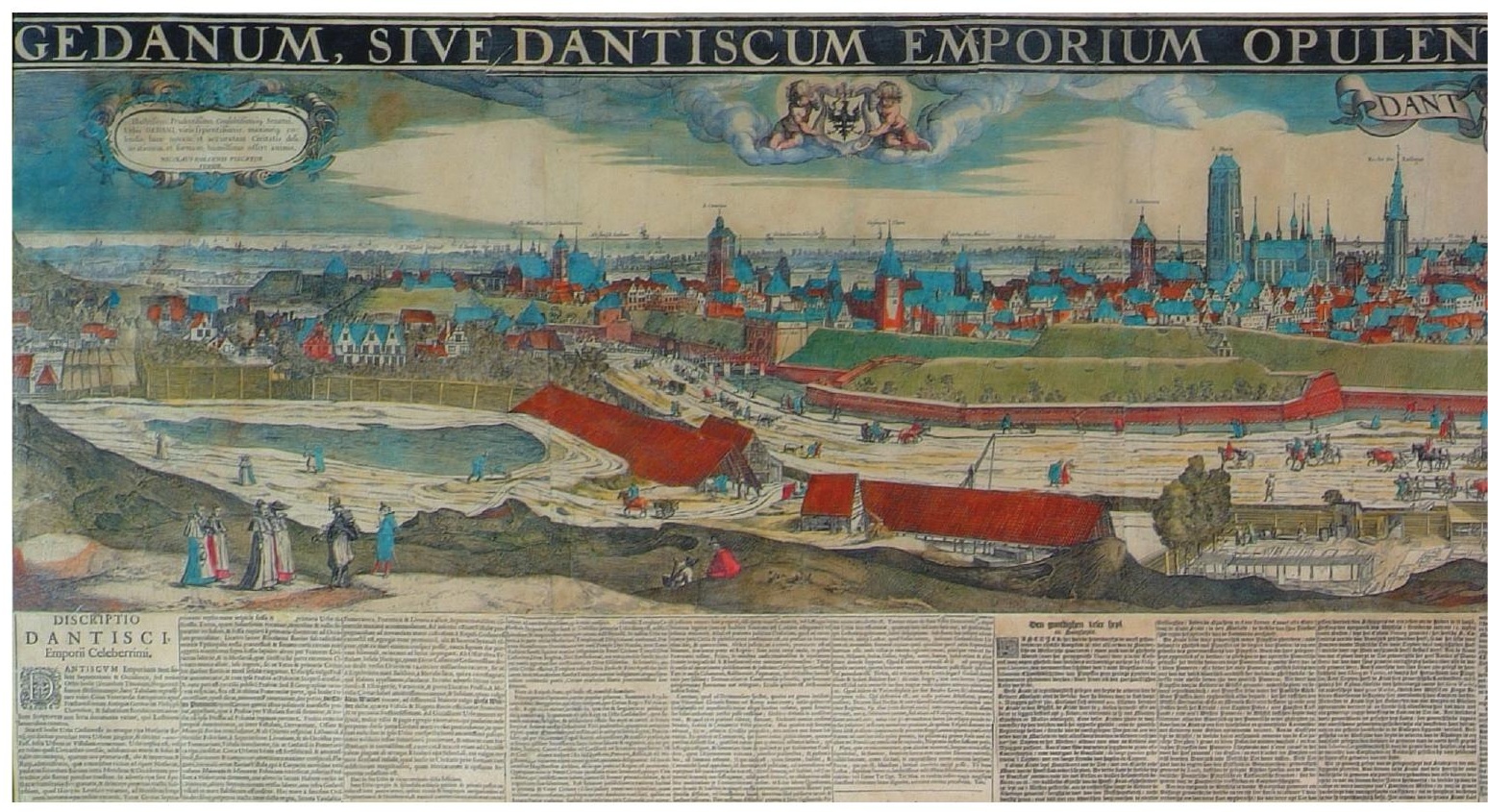

Abb. 7. J.C. Visscher, Panorama von Danzig (nach Dickman), Radierung (Danzig, Nationalmuseum)

Jansz. Visscher den Verlag des Vaters übernommen hatte. Dass das Monogramm Dickmans fehlt, mag seinen einfachen Grund darin haben, dass Visscher die Motive am Himmel, wo sich dessen Signatur befand, ausradiert hat. Visschers Danzig-Panorama unterscheidet sich in einigen Punkten von der Mehrzahl der in Amsterdam produzierten Städteansichten. Mit einer Länge von 213,3 cm ist es kleiner als Dickmans Original und damit den üblichen Maßen der Amsterdamer Drucke angepasst. Es ist jedoch nicht, wie diese, von vier, sondern von sechs Platten gedruckt worden und erinnert damit an Dickmans Vedute, die von sieben Platten gedruckt wurde. Die Breite der von Dickman und Visscher verwendeten Platten und die darauf dargestellten Abschnitte stimmen von geringfügigen Abweichungen abgesehen überein. Dass Visschers Vedute kleiner ist als Dickmans, kommt daher, dass die Darstellung vom Dorf Schottland weggelassen worden ist. Das erklärt, warum die zwei Platten vom rechten Ende von Dickmans Panorama bei Visscher durch nur eine, relativ schmale ersetzt worden $\operatorname{sind}^{19}$. Beim Höhenmaß ist der Unterschied dagegen groß: $53,7 \mathrm{~cm}$ bei

19 Die unteren Breiten der von Dickman benutzten Platten betragen von links nach rechts: 40,0, 41,0, 40,5, 41,5, 39,5, 39,0 bzw. 16,5 cm. Die Platten wurden am unteren Rand gemessen, und die Maße sind nicht auf den Millimeter exakt. Ich danke Malin Joakimson, Kungl. Biblioteket, für diese Information. Die von Visscher verwendeten Platten haben, von links nach rechts, die Maße: 38,0, 40,5, 40,9, 40,8, 40,0 bzw. 13,1 cm. Diese Angaben nach Hollstein's Dutch and Flemish Etchings, c. 1450-1700, vol. 38, Nr. 132. 
Lars Olof und Dickmans Fähigkeit, die einzelnen Motive, ungeachtet der Präzision ihrer Larsson Wiedergabe, in der Gesamtheit der Darstellung aufgehen zu lassen, kommt dort, wie auch in den 14 Ansichten, überzeugend zum Ausdruck und verdient unsere Anerkennung. Zum Wert seines Werkes trägt schließlich die auch im europäischen Kontext relativ frühe Entstehung sowohl der 14 Ansichten als auch des großen Stadtpanoramas bei.

\section{Literatur}

Behringer Wolfgang, Roeck, Bernd, Das Bild der Stadt in der Neuzeit 1400-1800, München 1999.

Besing Thomas, Produktion und Publikum - Aspekte der Herstellung, Verbreitung und Rezeption frühneuzeitlicher Stadtdarstellungen [In:] Behringer und Roeck, S. 94-100.

Braun Georg, Hogenberg Frans, Städte der Welt. Gesamtausgabe der kolorierten Tafeln 1572-1617, Hg. Stephan Füssel, Köln 2017.

Collijn Isak, Magnus Gabriel de la Gardies samling af äldre stadsvyer och historiska planscher i Kungl. Biblioteket (Kungl. bibliotekets handlingar 35. Årsberättelse för år 1912), Stockholm 1915.

Cuny Georg, Danziger Kunst und Kultur im 16. und 17. Jahrhundert, Bd. 1, Frankfurt am Main 1910.

Curicke Reinhold, Der Stadt Danzig historische Beschreibung (1687), Hg. Siegfried Rosenberg, Hamburg 1979.

Ehrensvärd Ulla, Kokkonen Pellervo, Nurminen Juha, Mare Balticum. 2000 Jahre Geschichte der Ostsee, Helsinki 1996.

Hoff Bert van't, Grote Stadspanorama's, gegraveerd in Amsterdam sedert 1609, „Jaarboek van het Genootschap Amstelodamus“" 1955, 47, S. 81-131.

Hollstein's Dutch and Flemish Etchings, Engravings and Woodcuts, c. 1450-1700, Bd. 9., 1953.

Hollstein's Dutch and Flemish Etchings, Engravings and Woodcuts, c. 1450-1700, Bd. 38, 1991.

Hollstein's German Etchings, Engravings and Woodcuts, c. 1400-1700, Bd. 6, 1960.

Jäger Eckhard (Hg.), Ægidius Dickmann und das Danziger Stadtbild um 1617 (mit Einführung und Kommentaren von Ernst Bahr), Lüneburg 1979.

Lingenberg Heinz, „Der älteste Grundrissplan Danzigs - ein Werk Anton Möllers?“ [In:] Unser Danzig, Bd. 1-2, 1984.

Meyer Hans, Die Danziger Stadtvedute in ihrer künstlerischen Würdigung (Diss.), Königsberg 1934.

Simon Maria, Claes Jansz. Visscher (unpublizierte Diss. Freiburg i. Br. 1958; ein Ex. in der Bibliothek des Kunsthistorischen Instituts der CAU Kiel).

Von Danzig nach Riga. Ansichten, Stadtpläne und Landkarten von Ost- und Westpreußen, Danzig und dem Baltikum aus der graphischen Sammlung des Germanischen Nationalmuseums, Nürnberg o.J. 
Nieco ponad sto lat temu w Bibliotece Królewskiej w Sztokholmie odkryto Wielką Panoramę Gdańska z 1617 r. Praca o dumnie brzmiącym tytule: DANTISCUM SEPTENTRIONIS; OCCIDENTIS ET TOTIUS ORBIS HODIE EMPORIUM FREQUENTISSIMUM jest drukowaną z siedmiu płyt akwafortą o nietypowej wielkości $(53,7 \times 258 \mathrm{~cm})$. Grafika sygnowana jest „Aegidius Dickman Gedanensis fecit et excudebat 1617”, co oznacza, że autor w inskrypcji nazywa siebie gdańszczaninem, twórcą dzieła i jego wydawcą.

Nie są znane inne odbitki tej weduty. Zachowała się natomiast seria 14 Widoków Gdańska PRAECIPUOR LOCORUM ET AEDIFICIORUM QUAE IN URBE DANTISCANA VISUNTUR ADUMBRACIO, sygnowana przez Dickmana i datowana na 1617 r. Oba dzieła powstały prawdopodobnie w Gdańsku. Dickman wykonując tak dużą panoramę miasta, liczył zapewne na profity w przyszłości. Niestety, rok później na jego drodze stanął amsterdamski wydawca Pieter van der Keere, kopiując jego wedutę. Dickman, co wiemy ze źródeł, udał się w 1624 r. do Holandii, prawdopodobnie specjalnie po to, by przeciwstawić się takiemu procederowi. W Amsterdamie spotkał słynnego grafika i wydawcę - Claesa Jansza Visschera, i nawiazał z nim współpracę. W 1625 r. Visscher opublikował nowe wydanie 14 Widoków Gdańska Dickmana. Na ten sam rok może być datowana druga edycja Wielkiej Panoramy Gdańska wydana przez Visschera. Wcześniej uważano ją za kopię akwaforty Dickmana, ale wiele wskazuje na to, że została ona odbita, podobnie jak 14 Widoków Gdańska, z oryginalnych płyt Dickmana, a więc w porozumieniu z nim. Panorama wydana przez Visschera jest nieco mniejsza od pierwszej weduty Dickmana ze względu na fakt, że pominięto na niej wioskę Szkoty na południu miasta.

Głównym celem tekstu jest uchwycenie osobliwości Wielkiej Panoramy Gdańska autorstwa Dickmana na tle holenderskich widoków miast $\mathrm{z}$ tego okresu, przy jednoczesnym zwróceniu uwagi na jej niezwykłą oryginalność. Służą temu porównania z kopią van der Keere’a i edycją weduty opublikowaną przez Visschera. Zależało mi również na dokładniejszym zbadaniu niektórych, nieuwypuklonych wcześniej aspektów weduty, zwłaszcza w odniesieniu do sztafażu na pierwszym planie i alegorii w partii nieba.

Tłumaczenie Anna Sobecka 